\title{
Bifunctional ITO layer with a high resolution, surface nano-pattern for alignment and switching of LCs in device applications
}

\author{
Hyeon Su Jeong ${ }^{1,4}$, Hwan-Jin Jeon ${ }^{1,4}$, Yun Ho Kim², Moon Bee $\mathrm{Oh}^{3}$, Pankaj Kumar ${ }^{3}$, Shin-Woong Kang ${ }^{3}$ \\ and Hee-Tae Jung ${ }^{1}$
}

We describe a novel method for liquid crystal (LC) alignment using nano-patterns of electrically conductive indium-tin oxide (ITO) layers with high resolution $(c a<20 \mathrm{~nm})$ and high aspect ratio (ca 10), fabricated based on the secondary sputtering phenomenon. The ITO pattern developed in this manner not only provides high anchoring energy comparable to that of rubbed polyimides, but also maintains its low resistivity as an electrode. As a result, the patterned ITO can function as an electrode and alignment layer at the same time, which facilitates successful fabrication of bifunctional conductive alignment layer for LC devices. The LC cells fabricated using patterned ITO substrates show highly stable alignment of LCs over large area and good electro-optical responses. Moreover, systematic approach made by the precise control of pattern dimensions allows us to estimate a critical anchoring energy required for an effective LC alignment based on Berreman's theory.

NPG Asia Materials (2012) 4, e7; doi:10.1038/am.2012.12; published online 17 February 2012

Keywords: alignment; indium-tin oxide; ion bombardment; liquid crystal; lithography; nano-pattern

\section{INTRODUCTION}

Conventional liquid crystal (LC) electro-optical devices incorporate separate layers of a transparent electrode and an organic polymer film for driving devices and controlling LC alignment, respectively. To date, the most common method to align LCs is by mechanical rubbing of a thin polymer film ${ }^{1}$ coated on the surface of a transparent electrode. Although rubbing is a simple and inexpensive method, it has many intrinsic drawbacks such as generation of electrostatic charges, contamination from rubbing debris and high temperatures (up to $c a 250^{\circ} \mathrm{C}$ ). As alternatives to the rubbing process, noncontact methods such as photo-alignment ${ }^{2-5}$ and ion-beam irradiation ${ }^{6}$ have been used but they also have critical drawbacks associated with controlling the orientation of photoswitchs on the surface and low anchoring energies. ${ }^{7}$

Indium-tin oxide (ITO) has been widely used as an electrode for LC display because of its low electrical resistance and high transmittance in the visible range of the optical spectrum. ITO surface is much harder than the polymer surface. Therefore, conventional rubbing method can hardly deform the surface of ITO. There are a few efforts, such as atomic force microscopy (AFM) rubbing, ${ }^{8}$ plasma etching, ${ }^{9}$ surface relief grating ${ }^{10}$ and ion-beam irradiation, ${ }^{11}$ which deal with patterning the ITO layer. However, in most methods, the quality of ITO pattern is inferior to that of polymer pattern. More importantly, all of these ITO patterning methods do not achieve uniform high resolution and aspect ratio patterns over large areas without significant loss of electrical conductivity and optical transmittance, factors that are directly related to anchoring energies of LCs and energy efficiencies of devices.

In the study described below, a novel method has been developed for LC alignment. The technique uses high resolution $(c a 20 \mathrm{~nm})$ and a high aspect ratio ( $c a$ 10) nano-pattern of electrically conductive ITO, which are fabricated by employing a new patterning technique that relies on a secondary sputtering phenomenon (SSP). The ITO nanopattern developed in this manner not only has a high anchoring energy that is comparable to those of rubbed polyimides, ${ }^{12}$ but also it has a low resistivity as an electrode. As a result, the patterned ITO can function simultaneously as an electrode and an alignment layer, properties that facilitate the fabrication of bifunctional conductive alignment layer for LC devices. The LC cells, constructed using these nanometer scale patterns on the surfaces of ITO layers, display highly stable alignment of LCs and good electro-optical responses over large areas.

\section{MATERIALS AND METHODS}

Figure 1 illustrates the overall procedure utilized for creating large-area ITOs, highly periodic nanostructures with high resolution $(<20 \mathrm{~nm})$ and high aspect

\footnotetext{
${ }^{1}$ Department of Chemical and Biomolecular Engineering, Korea Advanced Institute of Science and Technology, Daejeon, Korea; ${ }^{2}$ Advanced Functional Materials Research Group, Korea Research Institute of Chemical Technology, Daejeon, Korea and ${ }^{3}$ Department of BIN Fusion Technology, Chonbuk National University, Jeonju, Korea

${ }^{4}$ These authors contributed equally to this work.

Correspondence: Professor S-W Kang, Department of BIN Fusion Technology, Chonbuk National University, Jeonju, Korea.

E-mail: swkang@jbnu.ac.kr

or Professor H-T Jung, Department of Chemical and Biomolecular Engineering, Korea Advanced Institute of Science and Technology, 373-1, Guseong-dong, Yuseong-gu, Daejeon 305-701, Korea.

E-mail: heetae@kaist.ac.kr

Received 8 November 2011; revised 14 December 2011; accepted 23 December 2011
} 
a

Pattern transfer from PDMS mold to PS b Removal of PS on bottom by RIE
C Formation of thin ITO walls by SSP

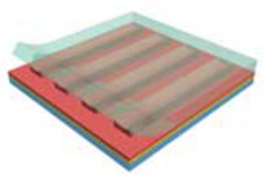

d Removal of PS template and resulting ITO pattern

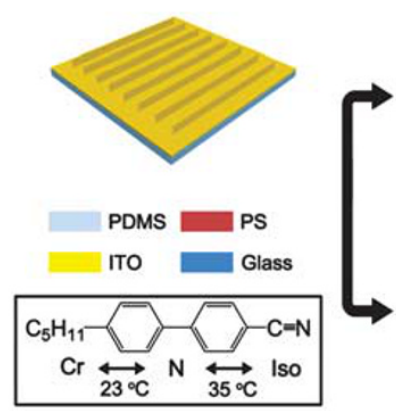

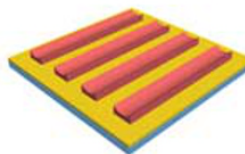

e1

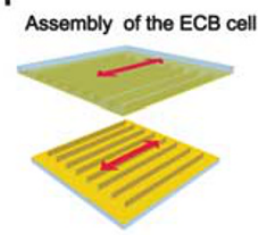

e2

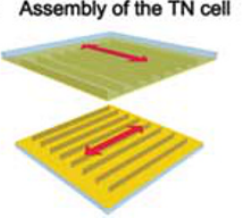

\section{.}

f
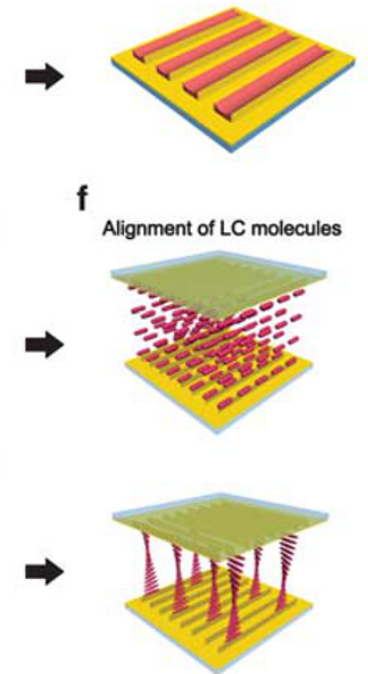

Figure 1 (a-d) Schematic illustration of the fabrication of ITO line-shaped patterns by using the secondary sputtering phenomenon and the use of the nanopatterned ITO layers as an alignment layer and electrode. (e) The ECB (e-1) and TN (e-2) mode cells fabricated for the study. (f) LC alignment of nematic $5 \mathrm{CB}$ in the ECB and TN cells. The molecular structure, phase sequence and transition temperature of $5 \mathrm{CB}$ are shown in the inset.

ratios (height to width ratio of $c a 10$ ), by using the $\mathrm{SSP},{ }^{13}$ and the fabrication of LC cells with the patterned ITO substrates in the absence of additional polymeric alignment layers. Commercial ITO glass substrates were prepared with a thickness of $180 \mathrm{~nm}$, resistivity of $c a 10 \Omega \square^{-1}$ and optical transparency of $96 \%$ at $550 \mathrm{~nm}$. Impurities on the ITO surface were removed using isopropyl alcohol through ultrasonic means. The ITO was subsequently dried by using a $\mathrm{N}_{2}$ gas stream. The strategy we have devised for LC alignment mainly relies on two key steps that involve attachment of a target material (that is, ITO) to sidewalls of pre-patterned polymers by means of Ar ion-beam bombardment (Figure 1c) and selective removal of the pre-patterned polymer (Figure 1d). As a result, a specific nano-structured pattern comprised of target materials remains on the flat ITO layer.

\section{Pattern fabrication on ITO surface}

To explore secondary sputtering on ITO, fabrication of the template began with spin coating a thin polystyrene (PS) film (6\% of PS (molecular weight $\left.=18000 \mathrm{~g} \mathrm{~mol}^{-1}\right)$ in anhydrous toluene). Then, the poly(dimethylsiloxane) (PDMS) mold was placed on the PS surface and heated above the glass transition temperature to drive PS polymer into the void spaces of the mold patterns by capillary action. This resulted in the formation of a negative copy of the mold (Figure 1a). Three kinds of PDMS molds were used, each containing strip patterns with a 1:1 width $(w)$ to spacing $(s)$ ratio $(w, s=200,500$ and $1000 \mathrm{~nm}$ ). The PS on the bottom was removed by utilizing reactive ion etching using a mixture of an $\mathrm{O}_{2}(40 \mathrm{sccm})$ and $\mathrm{CF}_{4}(60 \mathrm{sccm})$ plasma at a chamber pressure of $20 \mathrm{mTorr}$ and a power density of $80 \mathrm{~W}$. As a result of this process, only PS strip patterns comprising a 1:1 ratio of $w$ and $s$ without a bottom layer on the ITO glass were fabricated (Figure 1b). Then, the target ITO layer was selectively etched in the substrate plane and also partially in depth, and then transformed into a side of PS walls during an Ar+ ion bombardment process (Figure 1c). Finally, the pre-patterned PS film was removed by the second reactive ion etching process performed in the presence of $\mathrm{O}_{2}(100 \mathrm{sccm})$ to yield high-resolution ITO line patterns on top of the initial flat ITO layer over a $5 \times 5 \mathrm{~mm}^{2}$ area (Figure 1d). It should be noted that a broad range of feature sizes can be obtained by employing this technique by simply controlling the dimensions of the pre-existing polymer patterns and etching conditions.

\section{LC cell fabrication}

Electro-optic LC cells were prepared by assembling two substrates possessing ITO line patterns on their inner surfaces. As shown in Figure 1e, two types of
LC cells have been constructed, one having the same configuration as in the electrically controllable birefringence (ECB) mode and the other in the twistednematic (TN) mode, where the direction of the stripped ITO patterns on the top and bottom substrates are parallel and perpendicular to each other, respectively. The cell thickness was controlled by using microbead spacers (from 2 to $100 \mu \mathrm{m}$ ) and the cells were sealed by using a UV-curable adhesive (NOA 63, Norland Product Inc., Cranbury, NJ, USA). The cell gap was varied in the range $2.0-100.0 \mu \mathrm{m}$ in order to investigate the effects of cell thickness on LC alignment induced by the ITO patterns. The LC used in the study was $4^{\prime}$-pentyl-4cyanobiphenly (5CB, Aldrich Korea, Seoul, Korea), which is a single-component nematic (N) LC at room temperature. The molecular structure and phase transition temperatures of $\mathbf{5 C B}$ are shown in the inset of Figure 1. The fabricated empty cell was placed on a temperature-controlled hot stage (FP82HT Mettler Toledo Korea, Seoul, Korea), and the LC was loaded by employing capillary action at $40^{\circ} \mathrm{C}$ in the isotropic phase. After the filling process, the cell was cooled to room temperature at a rate of $2{ }^{\circ} \mathrm{Cmin}^{-1}$ and the state of LC alignment was probed using polarized optical microscopy (POM, Figure 1f).

\section{Electrical and optical properties of the patterned ITO substrates}

To measure the electrical conductance of the patterned substrate, a probe station (4200-SCS Keithley, Keithley Instruments, Inc., Cleveland, OH, USA) was used. This probe is comprised of contact electrodes ( $\mathrm{Au}$ ) that are deposited on both ends of patterned area by using e-beam evaporation. The electrical conductance was calculated by using the equation $o=G(l / t w)$, where $o$ is the electrical conductivity, $G$ is the electrical conductance obtained from the slope of the linear current-voltage curve, $l$ is the distance between two electrodes and $t$ is the thickness of the electrode. UV/Vis absorption spectroscopy (92-570, JASCO, Tokyo, Japan) was used to determine optical properties. The patterned ITO area was positioned in the path of light propagation and bare ITO glass was used as a references.

\section{RESULTS}

\section{Conductive alignment layer}

Figure 2a shows a scanning electron microscope (SEM) image of a highly periodic ITO line pattern. White lines in the SEM image correspond to thin ITO walls while relatively darker regions represent flat ITO surfaces. These images also serve to confirm that highly periodic ITO patterns were successfully formed over the entire surface area $(5 \mathrm{~mm} \times 5 \mathrm{~mm})$, indicating that the patterned area can be scaled 

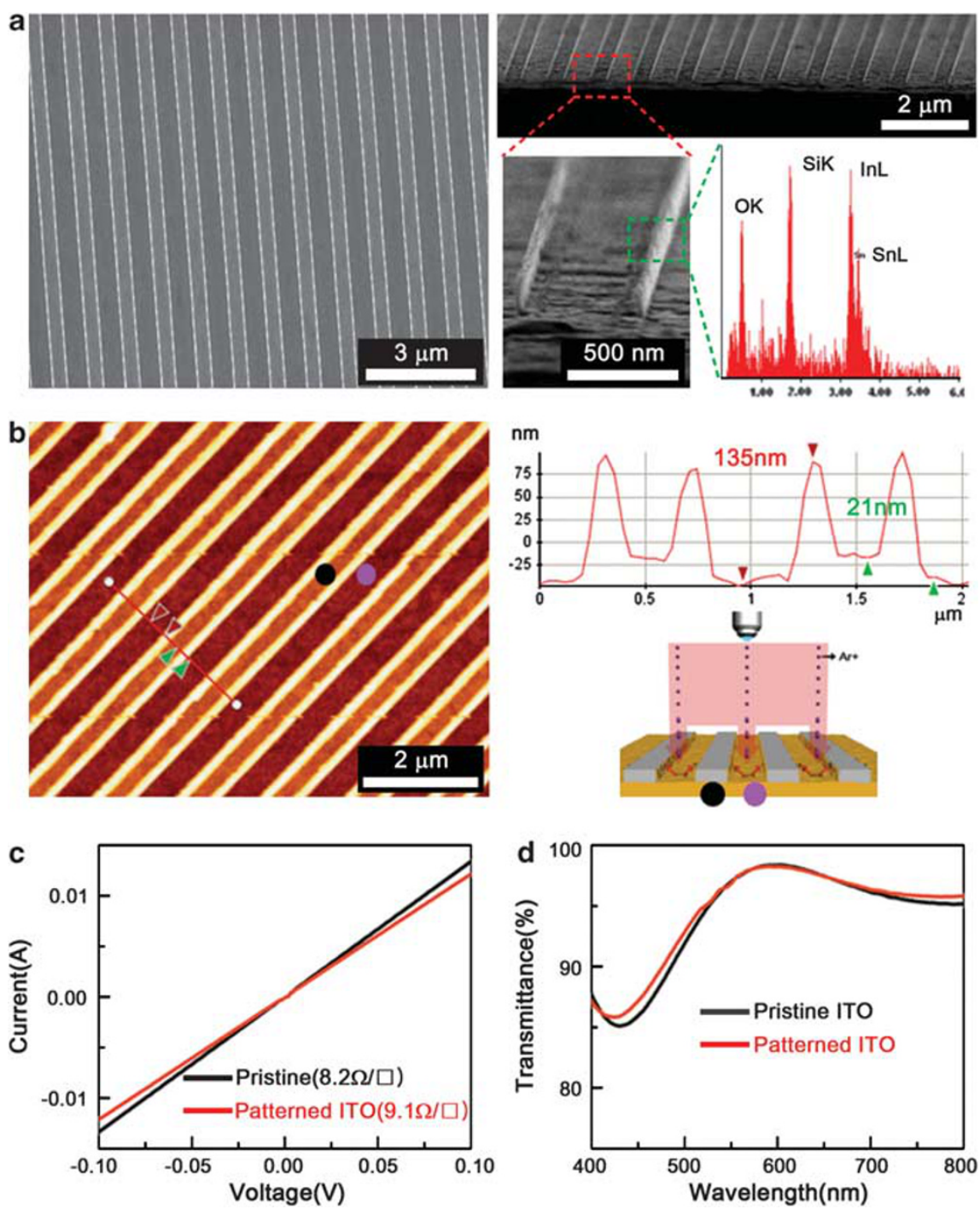

Figure 2 High-resolution ITO pattern with high aspect ratio over a large area without compromising electrical and optical properties. (a) Top and crosssectional SEM images of the ITO pattern with a dimension of 500-nm spacing. Magnified SEM image and energy dispersive X-ray spectroscopic (EDX) data of the ITO pattern are shown. (b) AFM image of the ITO pattern. The line-cut analysis shows a height (red triangle, $135 \mathrm{~nm}$ ) of the pattern and decreased thickness of the ITO layer (green triangle, $21 \mathrm{~nm}$ ). The partially eroded area during ion bombardment is marked by a purple dot while a black dot, previously occupied by the PS, represents the primitive flat ITO layer that is not affected by ion bombardment. (c) Current-voltage curves of pristine and patterned ITO substrates. (d) Optical transmittance spectra of pristine and patterned ITO substrates.

up if a larger mask is used. A slanting view of the SEM images clearly reveals that narrow lines of ITO are well developed and regularly aligned on the flat ITO layer over a large area (Figure 2a). The spacing between adjacent lines is found to be $c a 500 \mathrm{~nm}$, which is consistent with the spacing of a PDMS master mold that has a $500 \mathrm{~nm}$ width and spacing dimensions. However, the width of ITO line is observed to be ca $20 \mathrm{~nm}$, a value that is completely different from that of PS stripes $(500 \mathrm{~nm})$ replicated from PDMS mold. This difference is attributed to unique pattern formation caused by using the SSP. In this method, the sidewalls of the PS stripes act as supporters for the target material (ITO) to be attached and assembled as a thin wall during the ion bombardment process. ${ }^{13}$ The maximum aspect ratio was $\sim 18$ based on measurement of $11.8 \mathrm{~nm}$ width and $210 \mathrm{~nm}$ height of the ITO wall (Supplementary Figure S1). The ITO lines are likely to be stable in the range of aspect ratio of 10-13, and they tend to bend in higher aspect ratio. For this reason, we did not mention such a high aspect ratio $(>10)$. Energy dispersive X-ray spectroscopy was employed to verify that patterns are formed on the ITO. The energy dispersive $\mathrm{X}$-ray data show the existence of higher signals associated with indium $(\mathrm{In})$, tin $(\mathrm{Sn})$ and oxygen $(\mathrm{O})$, confirming that the lines are composed only of ITO.

Observations show that formation of the high resolution and aspect ratio ITO line pattern does not have diminishing effects on electrical and optical properties of the overall ITO layer, which are critical for simultaneous functioning of the patterned ITO as an electrode and alignment layer. Topological analysis of the line-cut profile connecting pairs of dots in the AFM image (red line in the image) shows the existence of highly uniform and regular thin walls that have a height of ca $135 \mathrm{~nm}$ (red triangles) (Figure 2b). The resulting aspect ratio is $c a 7$. It should be noted that the line width in the AFM image is broader 
than the actual width due to the nearly vertical drops of sidewall and fast scan rate of AFM tip. The decreased thickness of a primitive flat ITO layer can be easily seen by comparing the heights of the two flat areas marked by the black and purple dots in Figure 2b. As the ITO layer was selectively protected during ion bombardment of the pre-patterned polymer (that is, PS), the striped area marked by the black dot in Figure $2 \mathrm{~b}$ retained its original thickness. In the purple dot area, however, the ITO layer was uniformly eroded by ion bombardment and, as a result, the thickness decreased depending on the experimental conditions used. Under the condition given, a $c a 21 \mathrm{~nm}$ thick ITO layer (green triangles) was eroded to create thin walls with a height of $135 \mathrm{~nm}$ and a width of $20 \mathrm{~nm}$.

The conductivity of the ITO layer containing a nano-pattern on its surface is not greatly different from that of the pristine ITO layer owing to the only small decrease in thickness that occurs during bombardment. The current-voltage plots displayed in Figure $2 \mathrm{c}$ show that the electrical conductance of the patterned ITO layer is decreased slightly in comparison with that of the pristine counterpart. Analysis of the plots demonstrates that the electrical conductivity of the pristine ITO is $92850 \mathrm{~s} \mathrm{~m}^{-1}$ while that of patterned ITO is $89340 \mathrm{~s} \mathrm{~m}^{-1}$. The sheet resistance (inset in Figure 2c) of the ITO layers before and after patterning are $8.2 \Omega \square^{-1}$ and $9.1 \Omega \square^{-1}$, respectively. This observation indicates that a $c a 9 \%$ increase of sheet resistance has taken place upon patterning. In contrast, other previously described methods lead to significant increases in sheet resistance when the height of pattern increases. For example, the sheet resistance increases more than $100 \%$ after forming an ITO pattern with a height of $64 \mathrm{~nm}$ by utilizing the plasma etching method. ${ }^{13}$ As high transmittance in the visible range of the optical spectrum is important for display applications, we also examined the light transmittance of substrates before and after ITO patterning. Inspection of the UV/Vis spectra (Figure 2d) of the material before and after patterning shows that very little change has taken place in the transmittance (ca $96 \%$ at $550 \mathrm{~nm}$ ). As a matter of fact, the patterned substrate exhibits a slightly enhanced transparency at wavelengths below 500 and above $700 \mathrm{~nm}$. The results show that high-resolution ITO line patterns with high aspect ratios without diminishing electrical and optical properties of the overall ITO layer are formed. The patterns constructed in this manner are essential for cells that have potential applications in LC devices. This is a consequence of the fact that the resolution, aspect ratio and damage of ITO layers are directly related to the respective optical properties of the devices, uniformity of LC alignment and electrical conductivities. ${ }^{14,15}$

\section{Uniform alignment of LCs by the ITO pattern}

Nematic LC, 5CB, was introduced into cells having a $2-\mu \mathrm{m}$ gap in the isotropic phase. The cells were inspected by using a POM (LV-100POL, Nikon Korea, Seoul, Korea) equipped with a rotational stage in the plane perpendicular to the direction of light propagation. ${ }^{16}$ Figures $3 \mathrm{a}$ and $\mathrm{b}$ show wide-view POM images of the ECB cell, in which both substrates possess ITO patterns with a $20-\mathrm{nm}$ line width, $100 \mathrm{~nm}$ height and $500-\mathrm{nm}$ period in a $5 \times 5 \mathrm{~mm}$ area (see Supplementary Information, Supplementary Figure S2a). The differences that exist in optical textures between areas with and without the topographic pattern in the ITO layer are easily recognized. The central portion of the images highlighted by a yellow dotted square corresponds to the patterned area of the substrates. LC molecules on the patterned ITO surfaces are aligned uniformly parallel to the thin ITO line pattern (Figures $3 \mathrm{a}$ and $\mathrm{b}$ ), while LC molecules anchored on the non-patterned pristine ITO surface (outside of yellow dotted square) adopt a random azimuthal orientation as is typically observed for untreated solid surfaces. The POM image undergoes complete extinction when the striped ITO pattern is aligned either parallel or perpendicular to the polarizer or analyzer (Figure 3a). Fluctuations in light transmittance, caused by rotating the sample under the crossed polarizers, take place gradually in a sinusoidal fashion and reach a maximum value at $45^{\circ}$ (Figure $3 \mathrm{~b}$ ). ${ }^{17}$ The observations indicate that the LC molecules are oriented in a uniform and uniaxial manner (that is, nematic director) in the plane of the substrates.

POM images, taken with a quarter-wave retardation plate at different sample orientations, further demonstrate the azimuthal orientation of the director with respect to the ITO pattern (Figures $3 \mathrm{c}-\mathrm{f})$. Addition and subtraction of the total retardation created by placing the slow axis of the retarder in a parallel (Figure $3 \mathrm{e}$ ) and transverse (Figure 3f) direction with respect to the line pattern of ITO unambiguously demonstrate that the nematic director aligns along the thin ITO lines rather than in a transverse manner. We also substantiated by experiments that the LC aligning capability of nano-patterned ITO surfaces functions properly in a wide range of cell gap, at least, up to $100 \mu \mathrm{m}$ (the maximum thickness testified in our experiments, Supplementary Figure S3).

\section{Electro-optical responses}

POM image of the LC cell with a TN configuration were also recorded (Figure 4a). Two substrates, having a $5 \times 5 \mathrm{~mm}$ ITO pattern (see Supplementary Figure S2c) with a $20 \mathrm{~nm}$ width, $125 \mathrm{~nm}$ height and $500 \mathrm{~nm}$ spacing between lines, were orthogonally assembled in the cell. A non-uniform, random orientation of the director is observed in the non-patterned regions. However, the central area containing the topographically patterned ITO surface displays a uniform white color, which shows that the Mauguin's condition of the TN cell is attained. The electro-optic response of the TN cell to a square-wave electric field at $120 \mathrm{~Hz}$ normal to the substrate is shown in Figure $4 \mathrm{~b}$. The insets in this figure are POM images observed at $0 \mathrm{~V}$ and $2.5 \mathrm{~V}$. The observations show that, as the applied voltage increases, the LC changes its orientation from a twisted to homeotropic state, which results in a decrease of optical transmittance. The observations are typical V-T characteristics of a normally white mode TN cell, as are observed for a conventional cell and one containing a rubbed polyimide surfaces. The threshold voltage was found to be $c a 0.6 \mathrm{~V}$, and the voltage rise and fall times of the cell were determined to be $c a$ 1.6 and $2.4 \mathrm{~ms}$, respectively. The new TN cells were found to be quite durable and stable, maintaining their electro-optical responses over weeks of operation.

\section{DISCUSSION}

The results arising from the optical and electro-optical experiments carried out with the ECB and TN cells confirm that the nematic LCs can be unidirectionally aligned on the nano-patterned ITO surfaces. The mechanism for this phenomenon was proposed in 1971 by Berreman, ${ }^{15}$ and probed later using experiments with materials containing topographically anisotropic surfaces. ${ }^{18-24}$ In these cases, the driving force for LC alignment arises mainly from physical interactions between the surface and the LC molecules. The LC director is preferentially aligned along the direction of low roughness so that the total elastic free energy of the LC is minimized. ${ }^{25}$ As the flat and patterned surfaces in the cells we created are also composed of the ITO, the main difference lies in the topography of their surfaces. Therefore, we conjecture that the main driving force behind LC alignment on the patterned ITO surface is associated with the physical rather than the chemical anisotropy of the surface. It appears that the morphological anisotropy of the surface and the resultant physical 

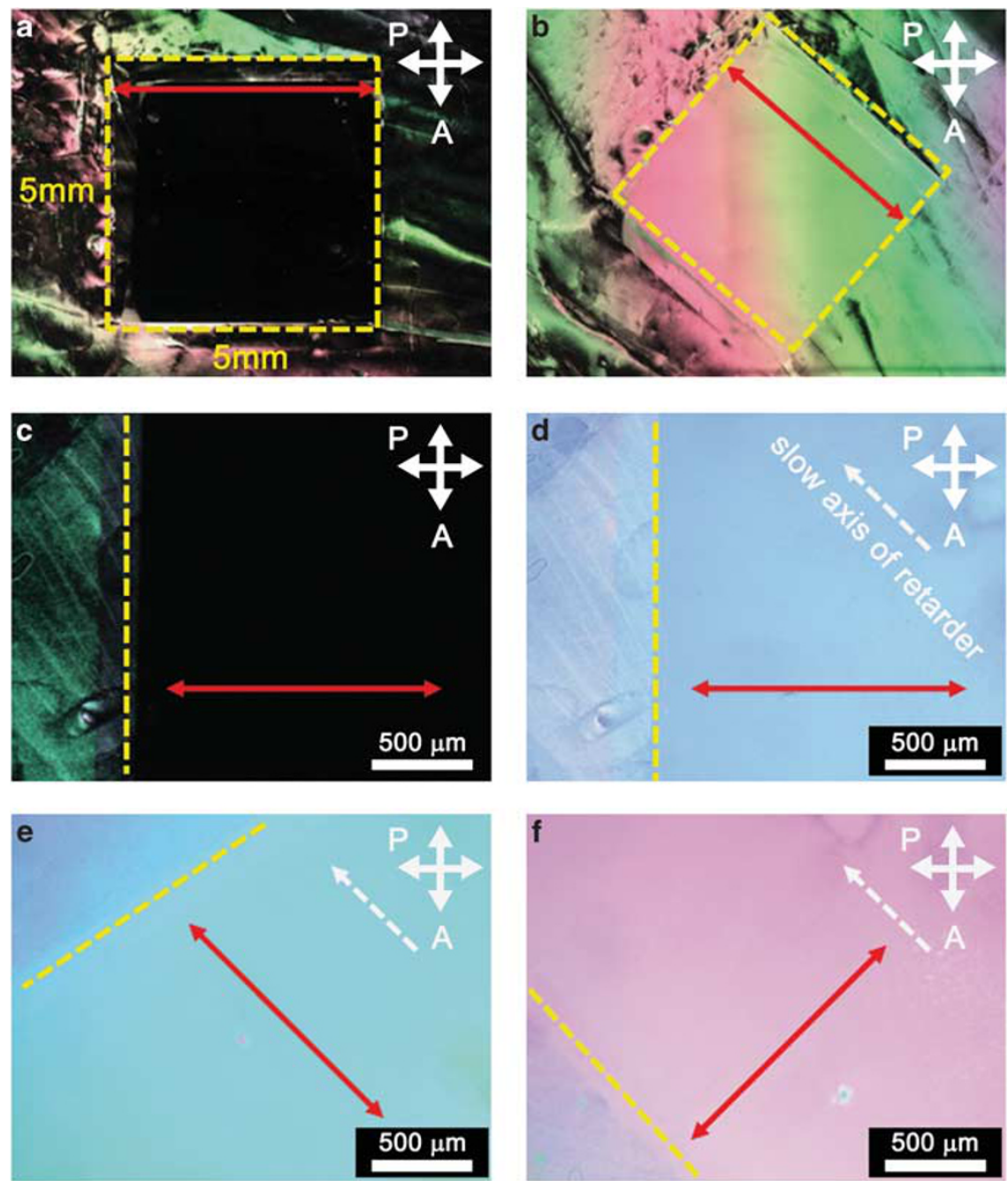

Figure 3 Uniform alignment of LCs by the ITO pattern. The ECB cell was constructed by using two ITO substrates, both possessing line-shaped patterns with $20 \mathrm{~nm}$ width, $98 \mathrm{~nm}$ height and $500 \mathrm{~nm}$ period over $5 \times 5 \mathrm{~mm}^{2}$ area. The borders are marked by dotted lines in yellow. The red arrows represent the direction of the ITO patterns. (a-b) Wide-view POM images for the directions of pattern (a) parallel and (b) $45^{\circ}$ to polarizer. (c-f) Variation of optical retardation observed with different orientations of quarter-wave plate with respect to the ITO pattern (a slow axis of the retarder is marked by white dotted arrows): POM images (c) without and (d) with retarder for the direction of ITO patterns parallel to polarizer. (e) and (f) are the POM images for the direction of ITO patterns parallel and perpendicular to the slow axis of the retarder, respectively.
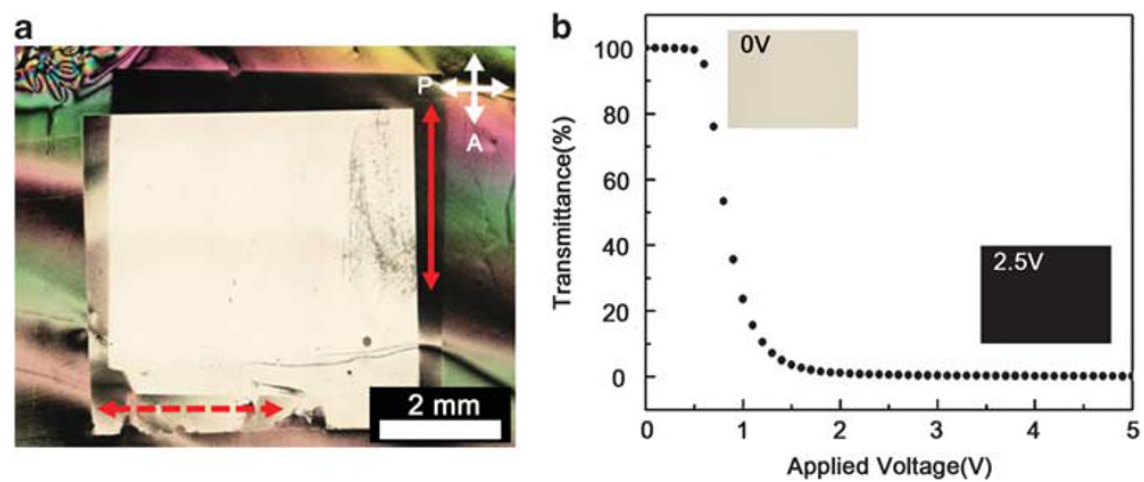

Figure 4 Electro-optical responses of the TN cell. The TN cell was comprised of two ITO-glass substrates that possess striped ITO patterns of $20 \mathrm{~nm}$ width, $125 \mathrm{~nm}$ height and $500 \mathrm{~nm}$ period over $5 \times 5 \mathrm{~mm}^{2}$ area. (a) Wide-view POM image of the TN cell measured in the depolarized condition. The solid and dotted arrows in red indicate the direction of the ITO lines on the top and bottom substrates, respectively. (b) The measured electro-optical response of the TN cell. The insets are the POM images of the cell at $O \mathrm{~V}$ and $2.5 \mathrm{~V}$. 
interaction with the LC molecules take place in a way to minimize the elastic free energy and that this promotes generation of a uniform azimuthal orientation of the LC director. Thus, in this case, the dimensions of the surface pattern and the elastic properties of the LCs combine to determine the characteristics of the LC alignment. According to Berreman's theory, the azimuthal anchoring energy of LC molecule on grooved surfaces is expressed as $W=2 \pi^{3} K A^{2} / \lambda^{3}$, where $A$ is the amplitude, $\lambda$ is the period of groove and $K$ is the twist Frank elastic constant of the LC. In the cells we created, the periods of the grooves were fixed at 200, 500 and $1000 \mathrm{~nm}$, as determined by spacings in the PDMS mold. Meanwhile, the height of the thin ITO lines was precisely controlled by the reactive ion etching conditions and concentration of the PS in toluene employed. The resulting aspect ratios range from $c a$ 1.5-10 (see Supplementary Figure S4).

Owing to the fact that this simple and versatile lithographic technique, which employs the SSP, enables accurate control of these parameters, a systematic investigation was carried out exploring how the characteristics of LC alignment are affected by the dimension of ITO patterns. With this goal in mind, ECB cells were fabricated and the quality of their LC alignments was examined by using POM analysis. Both the top and bottom substrates of the cell have thin ITO line pattern of the same periodicity and height. Figure 5 shows the variation of azimuthal anchoring energy as a function of height of the ITO lines for each period at a fixed width of $20 \mathrm{~nm}$. The periods of 200, 500 and $1000 \mathrm{~nm}$ are indicated by the black square, red circle and blue triangle, respectively. According to theory, the anchoring energies should increase with a decrease in period $(\lambda)$ and an increase in amplitude (A) (that is, height of ITO line). The maximum anchoring energy of patterned ITO is $3.96 \times 10^{-4} \mathrm{~N} \mathrm{~m}^{-1}$, a value that is comparable to those of conventional rubbed PIs $\left(10^{-4}-10^{-5} \mathrm{Nm}^{-1}\right)$ and surfaces rubbed by using AFM $\left(10^{-6} \mathrm{Nm}^{-1}\right){ }^{26}$ The minimum

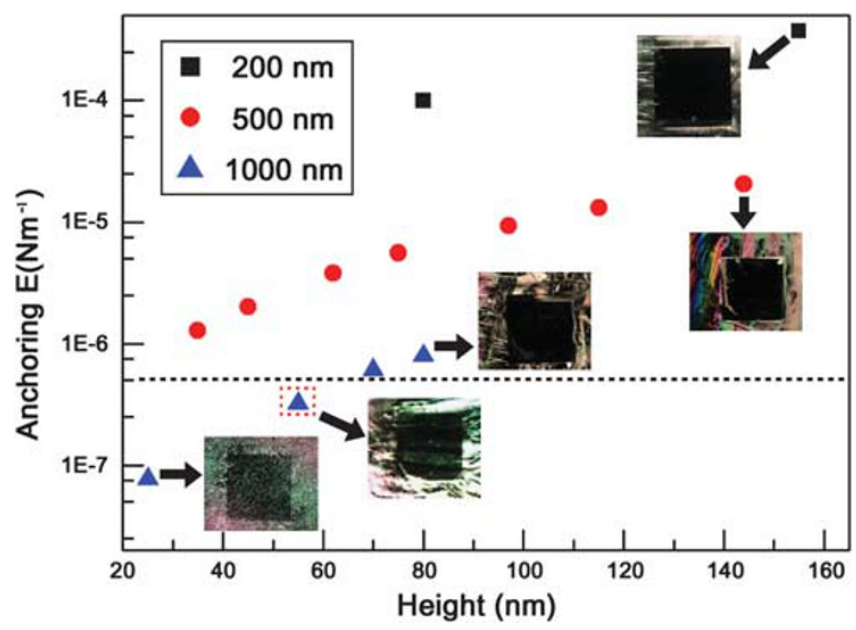

Figure 5 Critical anchoring energy for uniform LC alignment. Variation of azimuthal anchoring energy, estimated based on Berreman's theory, and corresponding POM images of the ECB cells as a function of height of thin ITO walls for each period at a fixed width of $20 \mathrm{~nm}$. The periods of 200 , 500 and $1000 \mathrm{~nm}$ are represented by the black square, red circle and blue triangle, respectively. Insets contain representative POM images of the ECB cell, corresponding to each point of the anchoring energy. The horizontal dashed line in the graph indicates a lower limit of azimuthal anchoring energy for a uniform director orientation of nematic LCs. The red dotted square for the 1000-nm period pattern represents the anchoring energy just below the limit, and the corresponding POM image shows a degradation of the LC alignment. anchoring energy is $7.75 \times 10^{-8} \mathrm{~N} \mathrm{~m}^{-1}$, which is 5100 times lower than the maximum value. Maximum and minimum anchoring energies were calculated using the line with $\lambda c a 200 \mathrm{~nm}$ and A ca $155 \mathrm{~nm}$ (Supplementary Figure S5) and the line with $\lambda \mathrm{ca} / 1000 \mathrm{~nm}$ and A ca $25 \mathrm{~nm}$ (Supplementary Figure S7), respectively.

The characteristics of LC alignment have been evaluated by using depolarized optical microscopy. The insets in Figure 5 are representative POM images of the ECB cells. Detailed information for each condition employed, including POM images with different orientations and AFM data of the substrates, are presented in Supporting Information (see Supplementary Figures S5-7). Except for the one with a 1000-nm periodicity, all other patterned ITO substrates display uniform and stable planar anchoring of the nematic $5 \mathrm{CB}$ along the parallel direction of the ITO pattern. However, a clear limit (represented by the horizontal dashed line in the graph) exists for a uniform director orientation throughout the entire active area. As shown in the inset of Figure 5, the cell constructed from the structured surface with a $51 \mathrm{~nm}$ height, $20 \mathrm{~nm}$ width and $1000 \mathrm{~nm}$ periodicity (corresponding anchoring energy $\mathrm{ca} 3.35 \times 10^{-7} \mathrm{~N} \mathrm{~m}^{-1}$ ) has a relatively poor uniformity. This observation indicates that in this case the anchoring strength of the surface is not sufficiently strong to constrain the LC molecules to a macroscopically uniform orientation.

The systematic approach involving precise control of the pattern dimensions has enabled us to estimate the critical anchoring energy that is required for effective LC alignment based on the Berreman's theory. Accordingly, when the estimated azimuthal anchoring energy is larger than $\mathrm{ca} 5 \times 10^{-7} \mathrm{~N} \mathrm{~m}^{-1}$ (corresponding to the pattern with a $64 \mathrm{~nm}$ height, $20 \mathrm{~nm}$ width and $1000 \mathrm{~nm}$ periodicity), the sample exhibits a uniform alignment of the LC molecules at the surfaces and, as a result, an ECB-type electro-optic cell is generated. The value of the azimuthal anchoring energy represents a minimal limit or guideline for the uniform LC alignment that arises from physically grooved surfaces.

\section{CONCLUSION}

In summary, the study described above has demonstrated that by using a lithographic technique that takes advantage of the SSP, a highquality surface pattern of ITO layer over a large area can be generated. In addition, this approach to patterning does not compromise the electrical and optical properties of the surface. These properties cause simultaneous creation of a transparent conductive layer and LC alignment layer. In addition, LC alignments on dimensionally controlled ITO patterns were systematically determined as a function of anchoring energy through employment of Berreman's theory. In this way, the critical anchoring energy for a uniform and stable alignment was defined. The approach described above for producing patterned ITO layers is not only cost effective but also leads to the capability of multi-directionally aligning LCs over very small dimensions, a result that is essential for constructing future LC display devices that have extraordinary performances and ultra-high resolutions.

\section{CONFLICT OF INTEREST}

The authors declare no conflict of interest.

\section{ACKNOWLEDGEMENTS}

This work was financially supported by grants from the National Research Laboratory Program (R0A-2007-000-20037-0), World Class University program (R32-2008-000-10142-0) and (R31-20029) funded by the Korea Ministry of Education, Science and Technology. We thank Professor M Srinivasarao for valuable discussions. 
1 Toney, M. F., Russel, T. P., Logan, J. A., Kikuchi, H., Sands, J. M. \& Kumar, S. K. Nearsurface alignment of polymers in rubbed films. Nature 374, 709-711 (1995).

2 Schadt, M., Seiberle, H. \& Schuster, A. Optical patterning of multidomain liquid-crystal displays with wide viewing angles. Nature 381, 212-215 (1996)

3 Schadt, M., Schmitt, K., Kozinkov, V. \& Chigrinov, V. Surface-induced parallel alignment of liquid crystals by linearly polymerized photopolymers. Jpn. J. Appl. Phys. 31, 2155-2164 (1992).

4 Park, B., Jung, Y., Choi, H. H., Hwang, H. K., Kim, Y., Lee, S., Jang, S. H., Kakimoto, M. \& Takezoe, H. Thermal and optical stabilities of photoisomerizable polyimide layers for nematic liquid crystal alignments. Jpn. J. Appl. Phys. 37, 5663-5668 (1998).

5 Yaroshchuk, O., Cada, L. G., Sonpatki, M. \& Chien, L. C. Liquid-crystal photoalignment using low-molecular-weight photo-cross-linkable composites. Appl. Phys. Lett. 79, 30-32 (2001)

6 Chaudhari, P., Lacey, J., Doyle, J., Galligan, E., Lien, S. C. A., Callegari, A., Hougham, G., Lang, N. D., Andry, P. S., John, R., Yang, K. H., Lu, M., Cai, C., Speidell, J., Purushothaman, S., Ritsko, J., Samant, M., Stöhr, J., Nakagawa, Y., Katoh, Y., Saitoh, Y., Sakai, K., Satoh, H., Odahara, S., Nakano, H., Nakagaki, J. \& Shiota, Y. Atomic-beam alignment of inorganic materials for liquid-crystal displays. Nature 411, 56-59 (2001).

7 Chigrinov, V., Muravski, A., Kwok, H. S., Takada, H., Akiyama, H. \& Takatsu, H. Anchoring properties of photoaligned azo-dye materials. Phys. Rev. E 68, 617021-617025 (2003)

8 Behdani, M., Rastegar, A., Keshmiri, S. H., Missat, S. I., Vlieg, E. \& Rasing, T. Submicron liquid crystal pixels on a nanopatterned indium tin oxide surface. App. Phys. Lett. 80, 4635 (2002)

9 Pang, C., Hwang, J., Park, K., Jung, D., Kim, H. \& Chae, H. Efficiency enhancement of polymer solar cells by patterning nanoscale indium tin oxide layer. J. Nanosci. Nanotechnol. 8, 5279-5283 (2008).

10 Yang, S., Yang, K., Niu, L., Nagarajan, R., Bian, S., Jain, A. K. \& Kumar, J. Patterning of substrates using surface relief structures on an azobenzene-functionalized polymer film. Adv. Mater. 16, 693-696 (2004).

11 Hwang, S., Jin, H. J., Yoon, T. H. \& Kim, J. C. Homogeneous alignment of liquid crystals on an ion-beam-exposed indium-tin-oxide surface without coating alignment layer. Jpn. J. Appl. Phys. 49, 121702 (2010).

12 Yokoyama, H. Surface anchoring of nematic liquid-crystals. Mol. Cryst. Liq. Cryst. 165 265-316 (1988)

13 Jeon, H. J., Kim, K. H., Baek, Y. K., Kim, D. W. \& Jung, H. T. New top-down approach for fabricating high-aspect-ratio complex nanostructures with $10 \mathrm{~nm}$ scale features. Nano Lett. 10, 3604-3610 (2010).
14 Takatoh, K., Hasegawa, M., Koden, M., Itoh, N., Hasegawa, R. \& Sakamoto, M. in Alignment Technologies and Applications of Liquid Crystal Devices. (eds Gray, G.W., Goodby, J.W.) Ch. 3 (Taylor \& Francis Inc., New York, 2005).

15 Berreman, D. W. Solid surface shape and the alignment of an adjacent nematic liquid crystal. Phys. Rev. Lett. 28, 1683-1686 (1972).

16 Kim, Y. H., Yoon, D. K., Jeong, H. S., Lavrentovich, O. D. \& Jung, H. T. Smectic liquid crystal defects for self-assembling of building blocks and their lithographic applications. Adv. Funct. Mater. 21, 610-627 (2011).

17 Yoon, D. K., Choi, M. C., Kim, Y. H., Kim, M. W., Lavrentovich, O. D. \& Jung, H. T. Internal structure visualization and lithographic use of periodic toroidal holes in liquid crystals. Nat. Mater. 6, 866-870 (2007).

$18 \mathrm{Nie}, \mathrm{Z}$. H. \& Kumacheva, E. Patterning surfaces with functional polymers. Nat. Mater. 7, 277-290 (2008).

19 Rüetschi, M., Grütter, P., Fünfschilling, J. \& Güntherodt, H. J. Creation of liquid crystal waveguides with scanning force microscopy. Science 265, 512-514 (1994).

20 Lin, R. \& Rogers, J. A. Molecular-scale soft imprint lithography for alignment layers in liquid crystal devices. Nano Lett. 7, 1613-1621 (2007).

21 Kim, S. R., Teixeira, A. I., Nealey, P. F., Wendt, A. E. \& Abbott, N. L. Fabrication of polymeric substrates with well-defined nanometer-scale topography and tailored surface chemistry. Adv. Mater. 14, 1468-1472 (2002).

$22 \mathrm{Kim}$, J. H., Yoneya, M. \& Yokoyama, H. Tristable nematic liquid-crystal device using micropatterned surface alignment. Nature 420, 159-162 (2002).

23 Lin, T. C., Huang, L. C., Huang, C. C. \& Chao, C. Y. Formation of self-assembled periodic grooves via thermal drawing lithography for alignment layers in liquid crystal devices. Soft Matter 7, 270-274 (2011).

24 Lin, T. C., Huang, L. C., Chou, T. R. \& Chao, C. Y. Alignment control of liquid crystal molecules using crack induced self-assembled grooves. Soft Matter 5, 3672-3676 (2009).

25 Kumar, S., Kim, J.- H. \& Shi, Y. What aligns liquid crystals on solid substrates? The role of surface roughness anisotropy. Phys. Rev. Lett. 94, 077803 (2005).

$26 \mathrm{Kim}$, J. H., Yoneya, M., Yamamoto, J. \& Yokoyama, H. Nano-rubbing of a liquid crystal alignment layer by an atomic force microscope: a detailed characterization. Nanotechnology 13, 133-137 (2002)

(c)

This work is licensed under the Creative Commons

Attribution-NonCommercial-No Derivative Works 3.0

Unported License. To view a copy of this license, visit http:// creativecommons.org/licenses/by-nc-nd/3.0/

Supplementary Information accompanies the paper on the NPG Asia Materials website (http://www.nature.com/am) 